\title{
Endoscopic ultrasound-guided through-the-needle microforceps biopsy improves diagnostic yield for pancreatic cystic lesions: a systematic review and meta-analysis
}

\section{(ㄷ)(1) $(-)$}

\author{
Authors \\ Thomas McCarty ${ }^{1}$, Tarun Rustagi ${ }^{2}$ \\ Institutions \\ 1 Division of Gastroenterology, Hepatology and \\ Endoscopy, Brigham and Women's Hospital, Harvard \\ Medical School, Boston, Massachusetts, United States \\ 2 Division of Gastroenterology and Hepatology, University \\ of New Mexico, Albuquerque, New Mexico, United \\ States
}

submitted 20.2.2020

accepted after revision 9.4.2020

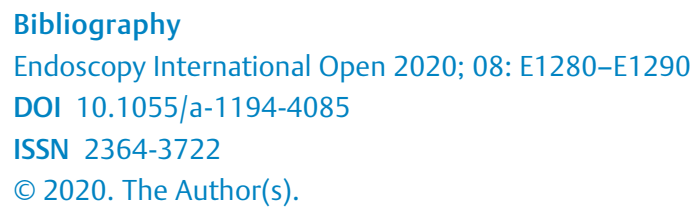
Commons Attribution-NonDerivative-NonCommercial License, permitting copying and reproduction so long as the original work is given appropriate credit. Contents may not be used for commecial purposes, or adapted, remixed, transformed or built upon. (https://creativecommons.org/licenses/by-nc-nd/4.0/)

Corresponding author

Tarun Rustagi, MD, Division of Gastroenterology and Hepatology, University of New Mexico, MSC10 5550, 1 University of New Mexico, Albuquerque NM 87131 Fax: +1-505-272-9751

tarunrustagi06@gmail.com

Supplementary material is available under https://doi.org/10.1055/a-1194-4085

\section{ABSTRACT}

Background and study aims Given variable diagnostic yield of endoscopic ultrasound (EUS)-guided fine-needle aspiration (FNA) for pancreatic cystic lesions (PCLs), a through-the-needle (TTN) microforceps biopsy device passed through a 19-gauge FNA needle has been devised to improve tissue sampling. This was a systematic review and meta-analysis to evaluate the feasibility, diagnostic yield, and safety of EUS-guided TTN microforceps biopsy for diagnosis of PCLs.

Methods Individualized searches were developed in accordance with PRISMA and MOOSE guidelines. This was a cumulative meta-analysis performed by calculating pooled proportions with rates estimated using random effects models. Measured outcomes included pooled technical success, diagnostic yield, accuracy, and procedure-associated adverse events (AEs) as well as comparison to conventional FNA.

Results Eleven studies ( $\mathrm{n}=518$ patients; mean age 64.13 \pm 5.83 years; $58.19 \%$ female) were included. Mean $P C L$ size was $33.39 \pm 3.72 \mathrm{~mm}$ with the pancreatic head/uncinate (35.50\%) being the most common location. A mean of $2.47 \pm 0.92$ forceps passes were performed with a mean of $2.79 \pm 0.81$ microbiopsies obtained per lesion. Pooled technical success was $97.12 \%\left(95 \% \mathrm{Cl}, 93.73-98.71 ; \mathrm{I}^{2}=\right.$ $34.49)$ with a diagnostic yield of $79.60 \%(95 \% \mathrm{Cl}, 72.62-$ $\left.85.16 ; \mathrm{I}^{2}=56.00\right)$, and accuracy of $82.76 \%[(95 \% \mathrm{Cl}$, $\left.77.80-86.80 ; I^{2}=0.00\right)$. The pooled serious adverse event rate was $1.08 \%\left(95 \% \mathrm{Cl}, 0.43-2.69 ; \mathrm{I}^{2}=0.00\right)$. Compared to conventional FNA, TTN microforceps biopsy resulted in significant improvement in diagnostic yield [OR 4.79 (95\% $\mathrm{Cl}$ : 1.52-15.06; $P=0.007)]$ and diagnostic accuracy [OR 8.69 (95\% Cl, 1.12-67.12; $P=0.038)$ ], respectively.

Conclusions EUS-guided TTN microforceps biopsy appears to be safe and effective for diagnosis of PCLs with improvement in diagnostic yield and accuracy when compared to FNA alone.

\section{Introduction}

Pancreatic cystic lesions ( $P C L s$ ) have become increasingly recognized with rising incidence due in part to significant improvements in high-resolution cross-sectional imaging [1,2].
While identification and frequency of PCLs has increased over the last decade, the decision to perform endoscopic ultrasound (EUS)-guided sampling for accurate diagnosis remains challenging. These cystic lesions can be classified as benign epithelial lesions, pre-malignant lesions, and malignant lesions as well as 
neuroendocrine neoplasms, or mesenchymal tumors according to the World Health Organization classification [3]. Cystic lesions in general may be even more broadly classified with a large differential diagnosis [4]. Diagnostic accuracy remains dependent on a number of factors, including lesion, location, inter-operator technique, skill, and experience, as well as the size or type of needle selected for tissue acquisition [5].

The need for accurate diagnosis is pivotal as some PCLs have pre-malignant or malignant potential. A key component of clinical management of pancreatic cysts is a reliable strategy to identify the small minority of cysts with early invasive cancer or high-grade dysplasia and to predict those cystic lesions that may develop into malignancy in the future [6]. Currently, EUSguided fine-needle aspiration (FNA) is a preferred sampling modality for PCLs; however, highly variable sensitivity and sensitivity as well as diagnostic yield exist given differing technique, lesion characteristic, and needle type utilized in clinical practice [6-8]. While FNA of the cyst wall may provide additional cytology material and increase the diagnostic yield for mucinous lesions, results remain highly variable [9]. Furthermore, distinguishing cystic wall nodules that are neoplastic (i. e., epithelial) from those that are non-neoplastic (i. e., mucinous) is critically important to properly risk stratify PCLs [10].

To improve the diagnostic yield of EUS-guided sampling for PCLs, a microforceps biopsy device has been recently designed with ability to pass through a standard 19-gauge FNA needle to perform through-the-needle (TTN) biopsy sampling of the cyst wall. As such, the primary aim of this study was to perform a structured systematic review and meta-analysis to evaluate the feasibility, effectiveness, and safety of EUS-guided TTN microforceps biopsy for diagnosis of PCLs. Secondary aims of this study were to compare diagnostic yield and accuracy of TTN microforceps biopsy to conventional FNA.

\section{Methods}

\section{Literature search}

Individualized literature search strategies were performed to in effort to identify full-text manuscripts that evaluated the safety and effectiveness of the TTN microforceps biopsy device for the diagnosis of PCLs. Systematic searches of PubMed, EMBASE, Web of Science, and the Cochrane Library databases were performed from available literature from inception through December 31, 2019. The following medical subject heading (MESH) terms included: microforceps biopsy. For articles related to microforceps biopsy, subject heading search terms and title and abstract were reviewed for: pancreatic cystic lesions (PCLs) and though-the-needle (TTN) biopsy.

All relevant English language full-text articles regardless of year of publication were included in this systematic review and meta-analysis. From the initial search results, duplicate articles were extracted, and then the titles and abstracts of all potentially relevant studies were screened for eligibility. The reference lists of studies of interest were then manually reviewed for additional articles by cross checking bibliographies as shown in the flow diagram. Two reviewers (TRM and TR) independently screened the titles and abstracts of all the articles ac- cording to predefined inclusion and exclusion criteria. In the case of studies with incomplete information, contact was attempted with the principal authors to obtain additional data.

\section{Study selection criteria}

This study was prospectively submitted in PROSPERO, an international database of prospectively registered systematic reviews in health and social care. The Preferred Reporting Items for Systematic Reviews and Meta-Analyses (PRISMA) statement outline and Meta-Analysis of Observational Studies in Epidemiology (MOOSE) reporting guidelines for reporting systematic reviews and meta-analyses was used to report findings - Appendix 1 and Appendix 2 [11, 12]. Full-text manuscripts and published abstracts were included in this analysis. Only human studies investigating the use of TTN microforceps biopsy device for the diagnosis of PCLs. All studies were required to report effectiveness or safety of the TTN microforceps biopsy device as this was the objective of this meta-analysis. A study was also excluded if deemed to have insufficient data, as were review articles, editorials, and correspondence letters that did not report independent data. Case series and reported studies with fewer than 10 patients were excluded to minimize selection bias. Multiple published work from similar authors was evaluated for overlapping enrollment times to preserve independence of observations.

\section{Outcome measures}

The primary outcome measurement in this study was the effectiveness and safety of the TTN microforceps biopsy device for the diagnosis of PCLs. Effectiveness and safety the microforceps biopsy device were measured by pooled technical success (i. e., the ability to perform successful TTN microforceps biopsy) and procedure-associated adverse events. All adverse events (AEs) were assessed based upon previously established criteria by the American Society of Gastrointestinal Endoscopy (ASGE) [13]. Additional testing characteristics of interest included diagnostic yield and diagnostic accuracy of the TTN microforceps device. Secondary outcomes, when possible, were to compare diagnostic yield and accuracy to traditional FNA. Other measured outcomes included baseline patient and lesion characteristics such as cyst location, size and presence of septations as well as procedural characteristics including number of biopsies and numbers of passes performed.

\section{Statistical analysis}

This systematic review and meta-analysis was performed by calculating pooled proportions. After appropriate studies were identified through systematic review, the individual study proportion was transformed into a quantity using the Freeman-Tukey variant of the arcsine square root transformed proportion. Then the pooled proportion was calculated as the back transform of the weighted mean of the transformed proportions, DerSimonian-Laird weights for the random effects model [14, 15]. The pooled rates were estimated using random effects models and presented as point estimates (rates) with $95 \%$ confidence intervals [16-18]. In contrast to fixed effect models, which are used to estimate a common effect, random effect 
models estimate an average effect, and the variability of the effects represented by their average may have clinical implications. All calculated $P$ values were 2 -sided, and $P<0.05$ was considered statistically significant. Tabular and graphical analyses were performing using Comprehensive Meta-Analysis software, version 3 (BioStat, Englewood, New Jersey, United States). Combined weighted proportions were determined by use of the Stata 15.0 software package (Stata Corp LP, College Station, Texas, United States).

\section{Risk of bias and quality assessment}

Risk of bias and quality of observational studies was evaluated using the Newcastle-Ottawa Quality Assessment Scale and JADAD score for quality of randomized trials $[19,20]$. In this study, high quality was defined as a Newcastle-Ottawa Quality Assessment Scale score of $\geq 4$ or a JADAD score of $\geq 3$. Two authors (TRM and TR) independently extracted data and assessed the risk of bias and study quality for each of the articles. Any disagreements were resolved by discussion and consensus between the two authors.

\section{Investigations of heterogeneity and prediction interval}

Heterogeneity was assessed for the individual meta-analyses using the chi squared test and the $\mathrm{I}^{2}$ statistic [17]. Significant heterogeneity was defined as $P<0.05$ using the Cochran $Q$ test or $I^{2}>50 \%$, with values $>50 \%$ indicating substantial heterogeneity. Further quantification of heterogeneity was categorized based upon $\mathrm{I}^{2}$ with values of $25 \%, 50 \%$, and $75 \%$ indicating low, moderate, and high amounts of heterogeneity, respectively. Given the use of random effects model to estimate average effect, a $95 \%$ prediction interval was calculated to determine the dispersion of effects and clearly illustrate heterogeneity in the calculated effect size $[16,21,22]$.

\section{Publication bias}

A funnel plot was created and visually inspected for asymmetry and quantitatively using Egger regression testing to assess for publication bias $[23,24]$. If evidence of publication bias, then the trim and fill method was used to correct for funnel plot asymmetry and provide an adjusted effect [25]. The classic fail-safe test was also applied to assess risk of bias across studies.

\section{Results}

\section{Baseline study and patient characteristics}

This meta-analysis included a total of 11 studies $(n=518)$ [2636]. A PRISMA flow chart of search results is shown in $\mathbf{F i g .} 1$. All studies were from 2018 to 2019, with 10 retrospective studies and one prospective study included. Three studies were multi-center in design with the remaining studies being singlecenter. Two published abstracts were included, and additional studies were full-text manuscripts. Baseline study, patient, and lesion characteristics are summarized in > Table 1 and Supplement Table 1. Fifty-eight percent of patients were female. Mean age of patients that underwent tissue sampling with TTN

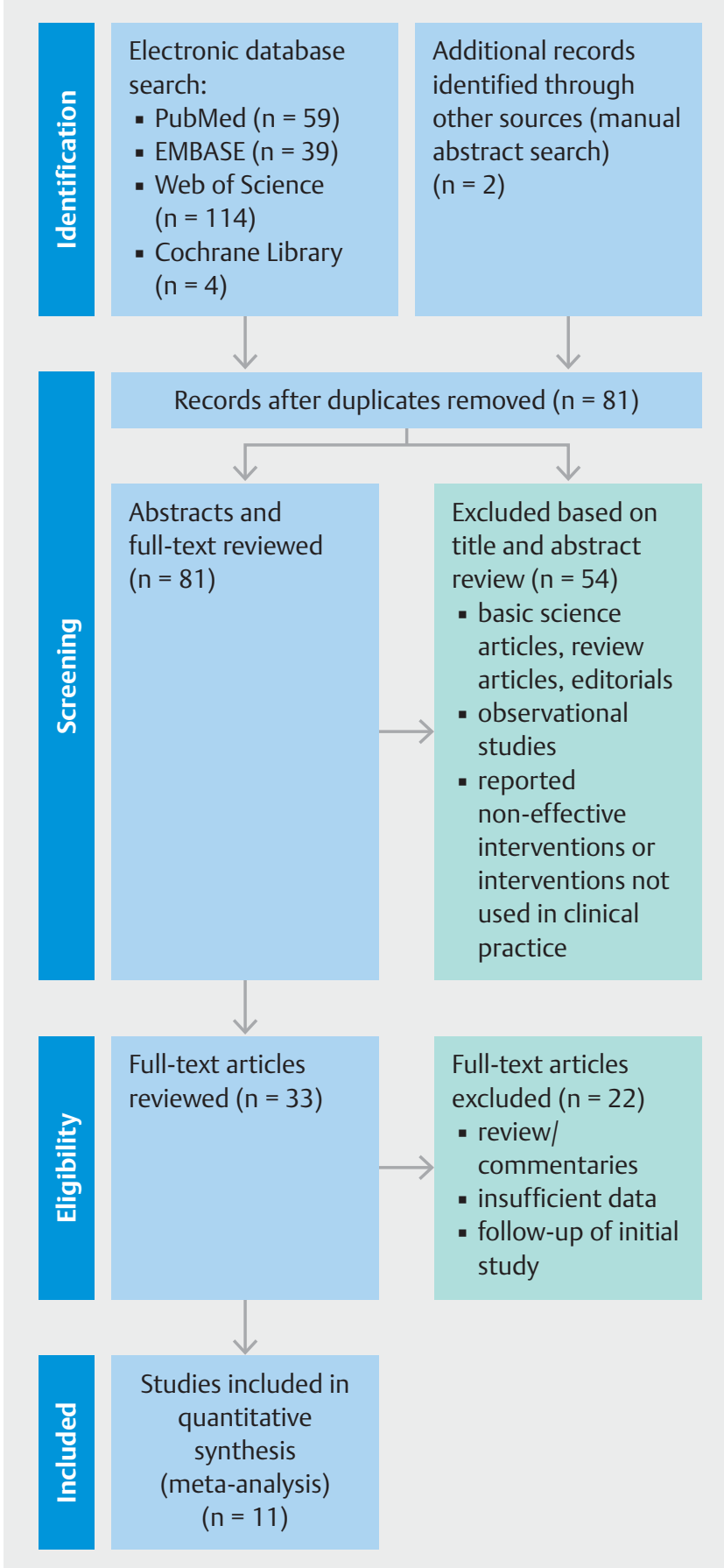

Fig. 1 Preferred Reporting Items for Systematic Reviews and Meta-Analyses (PRISMA) Flowchart of literature search results for through-the-needle microforceps biopsies of pancreatic cystic lesions. From: Moher D, Liberati A, Tetzlaff J et al. Preferred Reporting Items for Systematic Reviews and Meta-Analyses: The PRISMA Statement. PLoS Med 2000; 6: e1000097

microforceps was $64.13 \pm 5.83$ years. Mean size of patients' $\mathrm{PCL}$ was $33.39 \pm 3.72 \mathrm{~mm}$ with a variety of locations within the pancreas reported. Location in the head/uncinate process of the pancreas was the most common location of PCL (35.50\%), followed by body (32.47\%), tail (25.54\%), then neck (6.49\%). Of 


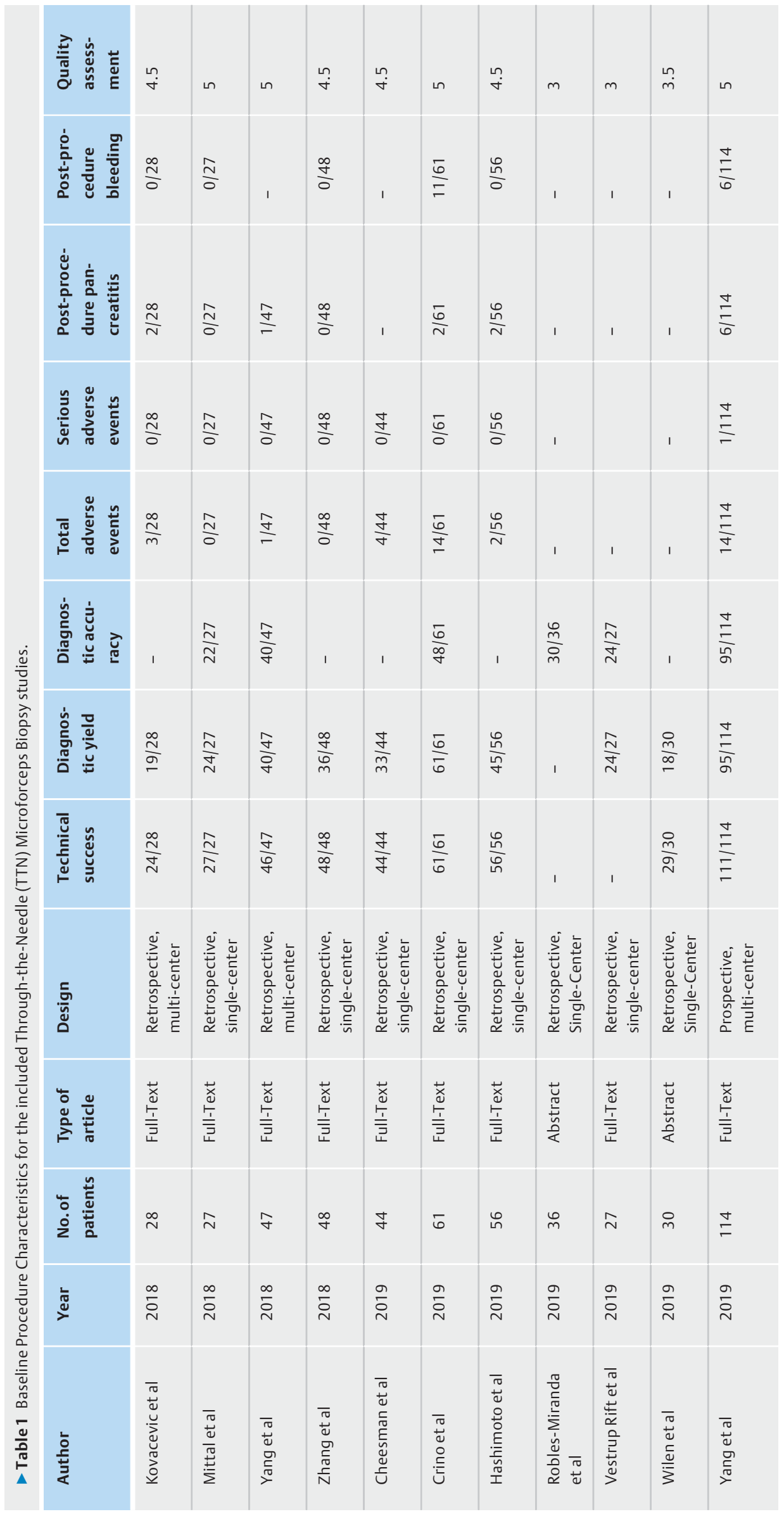


Technical success of Through-the-Needle Microforceps Biopsies

Study name

\begin{tabular}{lrrrr} 
& Event rate & Lower limit & Upper limit & Z-value \\
\hline Kovacevic et al. 2018 & 0.8571 & 0.6755 & 0.9453 & 3.317 \\
Mittal et al. 2018 & 0.9821 & 0.7704 & 0.9989 & 2.808 \\
Yang et al. 2018 & 0.9787 & 0.8638 & 0.9970 & 3.7877 \\
Zhang et al. 2018 & 0.9898 & 0.8568 & 0.9994 & 3.2183 \\
Cheesman et al. 2019 & 0.9889 & 0.8457 & 0.9993 & 3.1563 \\
Crino et al. 2019 & 0.9919 & 0.8838 & 0.9995 & 3.389 \\
Hashimoto et al. 2019 & 0.9912 & 0.8747 & 0.9995 & 3.328 \\
Wilen et al. 2019 & 0.9667 & 0.7980 & 0.9953 & 3.3107 \\
Yang et al. 2019 & 0.9737 & 0.9216 & 0.9915 & 6.171 \\
& & & & \\
& 0.9623 & 0.9335 & 0.9789 & 10.628
\end{tabular}

Statistics for each study

Z-value $\quad P$-value

0.0009

0.0009

0.0002

0.0013

0.0016

0.0007

0.0009

0.0009

0.0000

0.0000
Event rate and $95 \% \mathrm{Cl}$

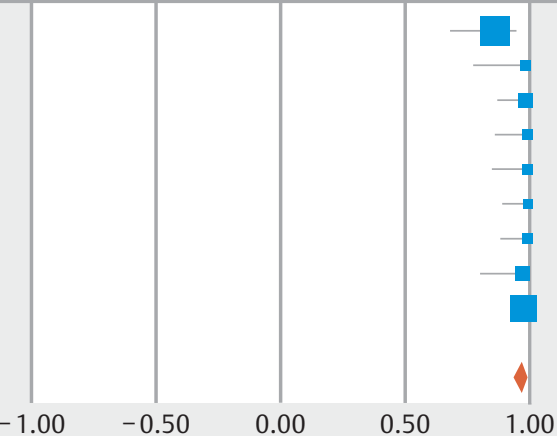

Diagnostic yield of Through-the-Needle Microforceps Biopsies

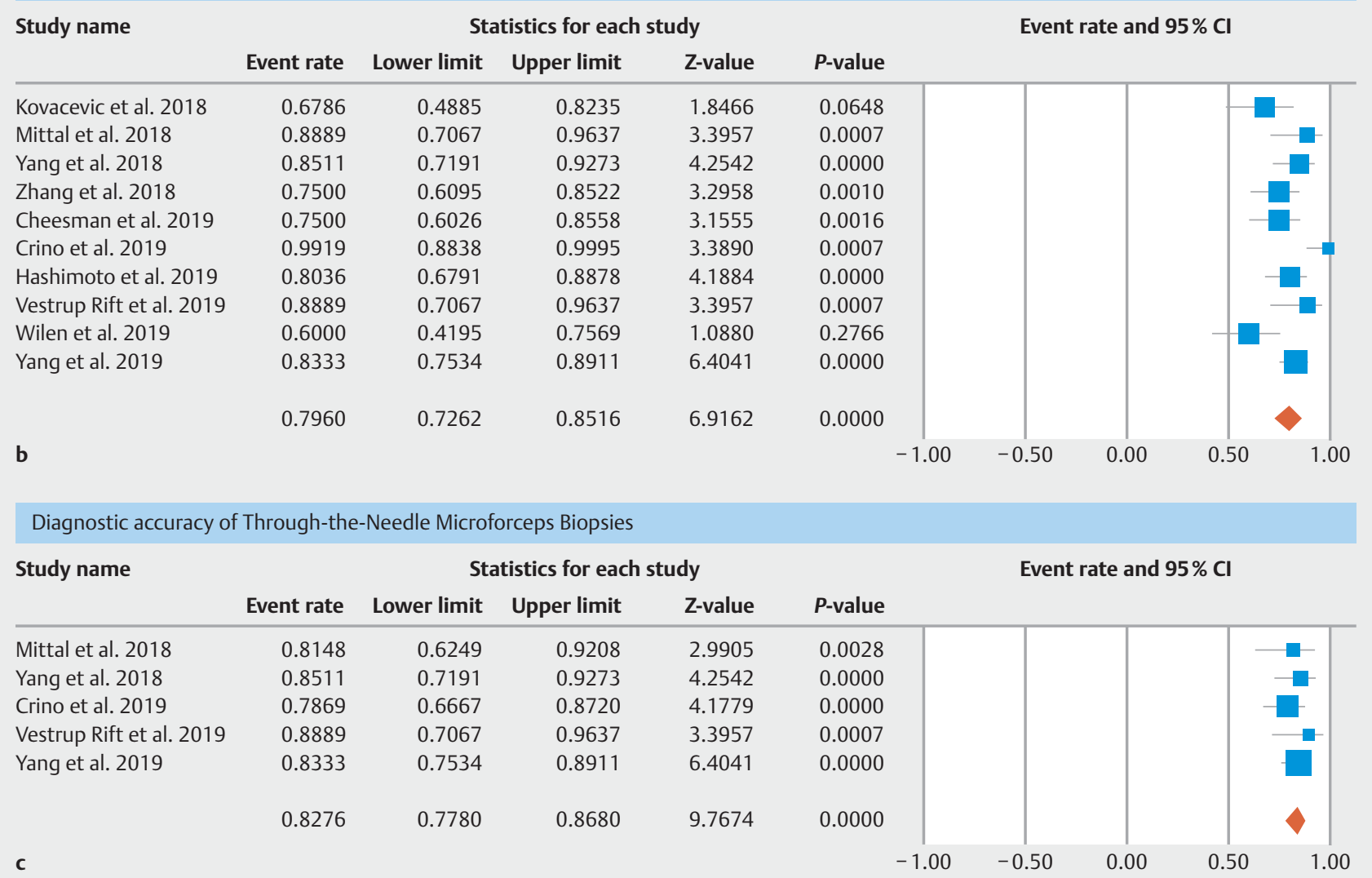

Fig. 2 a Pooled technical success of through-the-needle microforceps biopsies of pancreatic cystic lesions. b Pooled diagnostic yield of through-the-needle microforceps biopsies of pancreatic cystic lesions. c Pooled diagnostic accuracy of through-the-needle microforceps biopsies of pancreatic cystic lesions.

the three studies reporting EUS lesion characteristics, 38.36\% of lesions had a septated appearance.

\section{Procedural characteristics}

All included studies reported procedures using the Moray microforceps device [US Endoscopy, Ohio, United States]. The primary outcome of technical success of the TTN microforceps biopsy was reported in nine of 11 studies. The pooled technical success of the TTN microforceps biopsy device was $97.12 \%$ ( $95 \% \mathrm{Cl}, 93.73$ to $98.71 ; \mathrm{I}^{2}=34.49$; prediction interval 36.86 to 99.91) ( $>$ Fig. 2a). Diagnostic yield was also documented in all studies, with a pooled diagnostic yield of $79.60 \%(95 \% \mathrm{Cl}$, 72.62 to $85.16 ; I^{2}=56.00$; prediction interval 4.07 to 97.24 ). A mean of $2.47 \pm 0.92$ forceps passes were performed TTN 
with a mean of $2.79 \pm 0.81$ microbiopsies obtained per lesion ( $\triangleright$ Fig. 2b). Diagnostic accuracy was reported in 6 studies and found to be $82.76 \%\left[\left(95 \% \mathrm{Cl}, 77.80\right.\right.$ to $86.80 ; \mathrm{I}^{2}<0.01$; prediction interval 73.79 to 88.86 ) ( $\mathbf{F i g . 2 c}$ ). Only one included study reported mean procedure duration with a mean procedure time of $31.9 \pm 12.7 \mathrm{~min}$. The total pooled procedure-associated adverse event rate was $8.26 \%\left(95 \% \mathrm{Cl}, 4.39\right.$ to $15.02, \mathrm{I}^{2}$ =62.85; prediction interval-0.93 to 3.09) ( $\mathbf{F i g}$. 3a). The most common procedural associated adverse events were mild post-procedure pancreatitis and mild intracystic bleeding or hematoma managed conservatively in all patients [pooled rate of $3.94 \%\left(95 \% \mathrm{Cl}, 2.36\right.$ to $\left.6.48 ; \mathrm{I}^{2}<0.01\right)$ and $3.61 \%(95 \%$ $\mathrm{Cl}, 1.38$ to $9.09 ; \mathrm{I}^{2}=64.60$ ), respectively] ( $\mathbf{F i g . 3 b}$ ) and ( $\vee$ Fig.3c). Among 425 procedures performed, only one serious adverse event of moderate-severe pancreatitis with pseudocyst requiring endoscopic drainage was reported: pooled rate of $1.08 \%$ (95\% Cl: 0.43 to $2.69 ; \mathrm{I}^{2}<0.01$ ) ( Fig. 3d).

\section{Subgroup analyses}

All studies reported diagnostic yield of FNA alone compared to microforceps biopsy. When compared to sampling by conventional FNA, TTN microforceps biopsy resulted in significantly improved diagnostic yield [OR 4.79 (95\% Cl: 1.52 to 15.06);

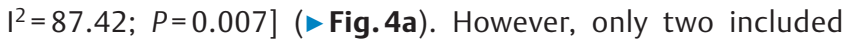
studies documented diagnostic accuracy of TTN microforceps compared to FNA alone.[27, 33,36] Based upon these two included studies, there was no significant difference in diagnostic accuracy noted between TTN microforceps biopsy versus FNA alone [OR $8.69(95 \% \mathrm{Cl}, 1.12$ to 67.12$) ; I^{2}=82.79 ; P=$ 0.038] ( Fig. 4b).

\section{Risk of bias assessment}

As only observational studies were included in this systematic review and meta-analysis, all studies were evaluated using the Newcastle-Ottawa Quality Assessment Scale. Quality assessment for each study demonstrated in $>$ Table 1 . All included studies were considered to be of high quality defined by Newcastle-Ottawa Quality Assessment Scale scores $\geq 4$. Visual inspection of the funnel plot demonstrated that smaller and statistically insignificant studies appeared to be missing likely due to publication bias - Fig.5a. With the Duval and Tweedie's trim and fill method, overall technical success was slightly decreased from $97 \%(95 \% \mathrm{Cl}, 93.73$ to 98.71$)$ to $95.39 \%(95 \% \mathrm{Cl}$, 90.58 to 97.80$)$ - Fig. 5b. Using the classic fail-safe test to assess for publication bias, it was determined it would take 266 non-significant studies to nullify the results of this analysis.

\section{Discussion}

Based upon the results of this systematic review and meta-analysis, EUS-guided TTN microforceps biopsy appear to be safe and effective for diagnosis of PCLs. Pooled technical success was $97.12 \%$ with a low serious adverse event rate of $1.08 \%$. Furthermore, the pooled diagnostic yield with the TTN device was $79.60 \%$ with a diagnostic accuracy of $82.76 \%$ - significantly better than EUS-guided FNA sampling alone.
Prevalence of PCLs has rapidly increased within the last few decades - with a prevalence of $8 \%$ among asymptomatic individuals [37]. Additional data has revealed that this incidence increases with age as well with need to differentiate non-neoplastic from neoplastic lesions [38]. While this has sparked an increased awareness of these lesions, the natural history of PCLs remains less clear, with optimal management still under debate [39]. It is important to emphasize that patients who are not surgical candidates should not undergo further evaluation of incidentally found pancreatic cysts, regardless of cyst size [4]. The same approach should generally be undertaken for lesions with low-risk of malignant transformation. However, when the diagnosis is unclear for indeterminant lesions or for lesions with high-risk characteristics, EUS-guided FNA with cyst fluid analysis should be considered $[4,10]$. Given the variable diagnostic accuracy of FNA, there remains a delicate balance between need to ensure appropriate treatment with surgical resection for neoplastic lesions, while safeguarding against over-treatment and undue procedures as well as proper surveillance $[10,40]$.

At present, conventional sampling with FNA is associated with suboptimal and highly variable diagnostic yield of PCLs [41]. FNA cytology of cyst fluid and routine cyst fluid analysis with CEA and amylase are non-diagnostic in substantial proportion of these lesions which continue to pose diagnostic dilemma [42-44]. To combat this, multiple alternative or complimentary sampling modalities have been proposed including the use of rapid on-site cytopathology examination (ROSE), addition of molecular analysis including GRAS and KRAS mutations, use of EUS-guided confocal laser endomicroscopy (nCLE), and TTN microforceps biopsy [45-49]. This wealth of diagnostic sampling options further underscores the challenge endoscopists face when evaluating PCLs. Prior to 2018, evidence-based guidelines on the management of PCLs were lacking $[7,8]$. In a joint initiative, European evidence-based guides on the management of pancreatic cystic neoplasms were established with aim to improve diagnosis and management [50]. Yet despite these guidelines, use of microforceps biopsies are not mentioned given limited evidence. In recent American College of Gastroenterology (ACG) guidelines, use of TTN microbiopsy forceps are noted but no current recommendation is provided-instead commenting that preliminary literature is promising, but larger, prospective, multi-center studies are required [4].

The first use of TTN direct intracystic biopsy was reported by Aparicio in 2010, during a pancreatic cystoscopy with a fiber optic probe passed through a 19 gauge EUS needle for two patients with cystic lesions in the head of the pancreas [51]. In this early study, mucinous lesions were accurately diagnosed though one patient was noted to develop a delayed adverse event of severe post-procedure pancreatitis. Later, Barresi and colleagues performed microforceps biopsies of one patient prior to development of a newer generation microforceps device (Moray microforceps) [52]. This device is comprised of microforceps which are $230 \mathrm{~cm}$ in length with a jaw opening width of $4.3 \mathrm{~mm}$ and a sheath of $0.8 \mathrm{~mm}$ in diameter that easily passes through a 19-gauge EUS-FNA needle [53]. A benefit to the 


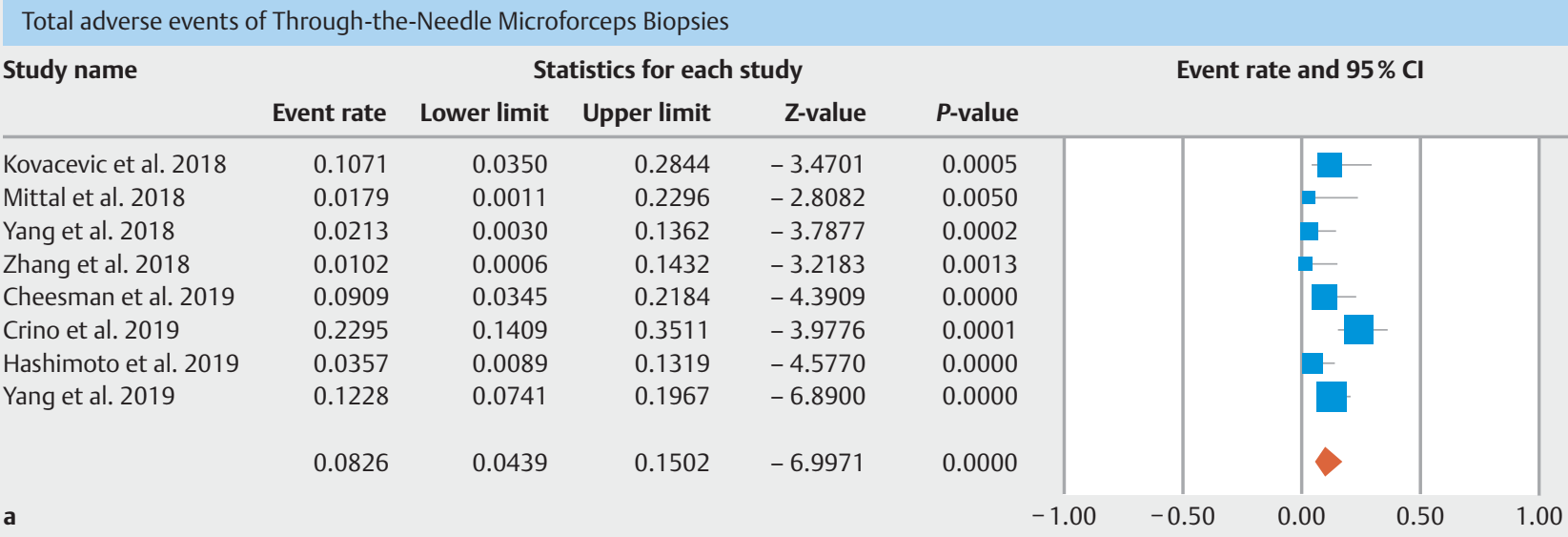

Post-procedure pancreatitis events of Through-the-Needle Microforceps Biopsies

\begin{tabular}{|c|c|c|c|c|c|c|c|c|c|c|}
\hline \multirow[t]{2}{*}{ Study name } & \multicolumn{5}{|c|}{ Statistics for each study } & \multicolumn{5}{|c|}{ Event rate and $95 \% \mathrm{Cl}$} \\
\hline & Event rate & Lower limit & Upper limit & Z-value & $P$-value & & & & & \\
\hline Kovacevic et al. 2018 & 0.0714 & 0.0179 & 0.2448 & -3.4954 & 0.0005 & & & $=$ & & \\
\hline Mittal et al. 2018 & 0.0179 & 0.0011 & 0.2296 & -2.8082 & 0.0050 & & & 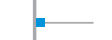 & & \\
\hline Yang et al. 2018 & 0.0213 & 0.0030 & 0.1362 & -3.7877 & 0.0002 & & & - & & \\
\hline Zhang et al. 2018 & 0.0102 & 0.0006 & 0.1432 & -3.2183 & 0.0013 & & & & & \\
\hline Cheesman et al. 2019 & 0.0111 & 0.0007 & 0.1543 & -3.1563 & 0.0016 & & & 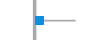 & & \\
\hline Crino et al. 2019 & 0.0328 & 0.0082 & 0.1218 & -4.7071 & 0.0000 & & & 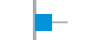 & & \\
\hline Hashimoto et al. 2019 & 0.0357 & 0.0089 & 0.1319 & -4.5770 & 0.0000 & & & $\square$ & & \\
\hline \multirow[t]{2}{*}{ Yang et al. 2019} & 0.0526 & 0.0238 & 0.1122 & -6.8911 & 0.0000 & & & & & \\
\hline & 0.0394 & 0.0236 & 0.0648 & -11.8964 & 0.0000 & & & $\downarrow$ & & \\
\hline b & & & & & & -1.00 & -0.50 & 0.00 & 0.50 & 1.00 \\
\hline \multicolumn{11}{|c|}{ Post-procedure bleeding events of Through-the-Needle Microforceps Biopsies } \\
\hline \multirow[t]{2}{*}{ Study name } & \multicolumn{5}{|c|}{ Statistics for each study } & \multirow{2}{*}{\multicolumn{5}{|c|}{ Event rate and $95 \% \mathrm{Cl}$}} \\
\hline & Event rate & Lower limit & Upper limit & Z-value & $P$-value & & & & & \\
\hline Kovacevic et al. 2018 & 0.0172 & 0.0011 & 0.2232 & -2.8341 & 0.0046 & & & - & & \\
\hline Mittal et al. 2018 & 0.0179 & 0.0011 & 0.2296 & -2.8082 & 0.0050 & & & 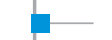 & & \\
\hline Zhang et al. 2018 & 0.0102 & 0.0006 & 0.1432 & -3.2183 & 0.0013 & & & - & & \\
\hline Crino et al. 2019 & 0.1803 & 0.1028 & 0.2970 & -4.5465 & 0.0000 & & & & & \\
\hline Hashimoto et al. 2019 & 0.0088 & 0.0005 & 0.1253 & -3.3281 & 0.0009 & & & 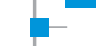 & & \\
\hline \multirow[t]{2}{*}{ Yang et al. 2019} & 0.0526 & 0.0238 & 0.1122 & -6.8911 & 0.0000 & & & - & & \\
\hline & 0.0414 & 0.0134 & 0.1209 & -5.3147 & 0.0000 & & & $>$ & & \\
\hline c & & & & & & -1.00 & -0.50 & 0.00 & 0.50 & 1.00 \\
\hline
\end{tabular}

Study name

Statistics for each study
Event rate Lower limit Upper limit Z-value P-value

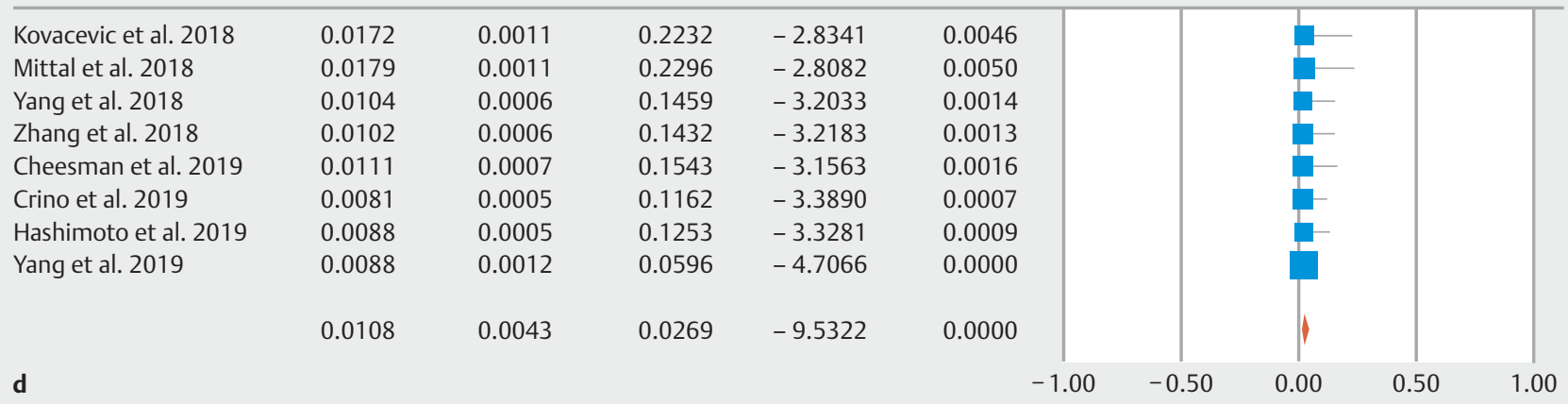

- Fig. 3 a Pooled adverse events of through-the-needle microforceps biopsies of pancreatic cystic lesions. b Post-procedure pancreatitis events of through-the-needle microforceps biopsies of pancreatic cystic lesions. c Post-procedure bleeding events of through-the-needle microforceps biopsies of pancreatic cystic lesions. $\mathbf{d}$ Serious adverse events of through-the-needle microforceps biopsies of pancreatic cystic lesions. 
Diagnostic yield of Through-the-Needle Microforceps Biopsies vs. Fine Needle Aspiration

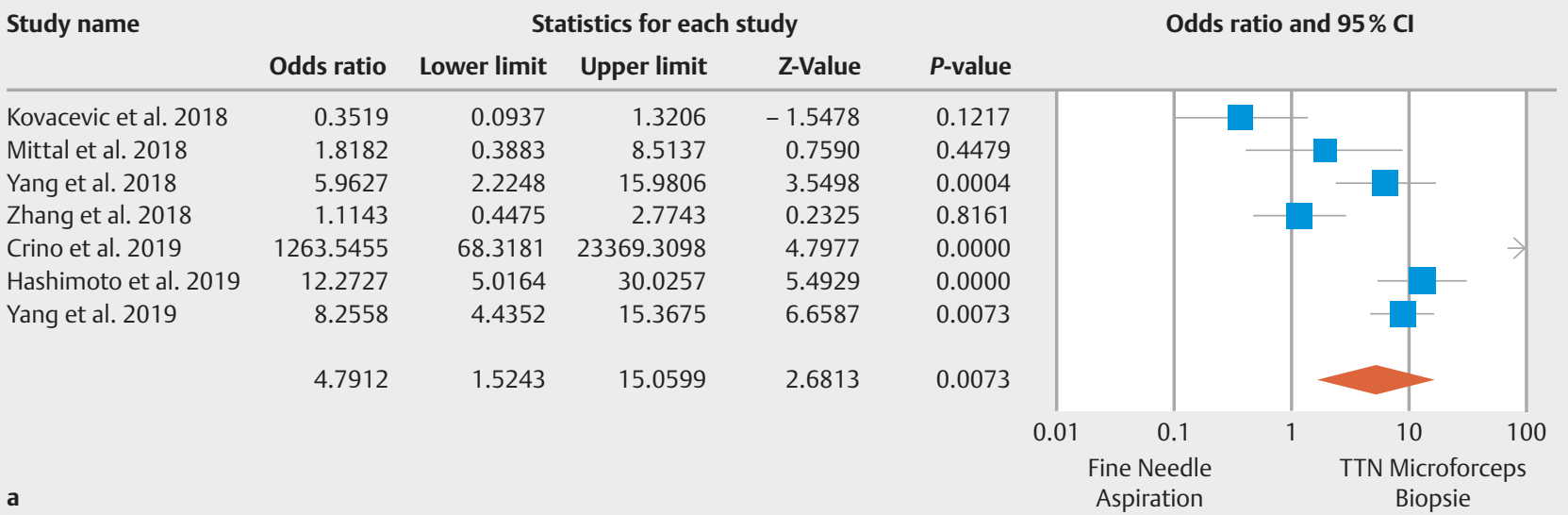

Diagnostic accuracy of Through-the-Needle Microforceps Biopsies vs. Fine Needle Aspiration

Study name

Statistics for each study

Odds ratio and $95 \% \mathrm{Cl}$

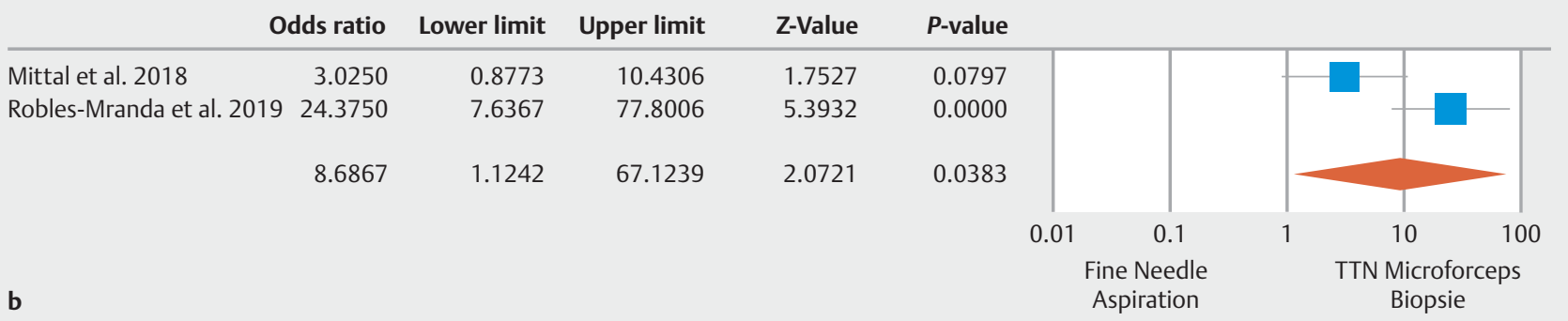

- Fig. 4 a Diagnostic yield of through-the-needle microforceps biopsies vs conventional fine needle aspiration for pancreatic cystic lesions. b Diagnostic accuracy of through-the-needle microforceps biopsies vs conventional fine-needle aspiration for pancreatic cystic lesions.

microforceps device is that this method of PCL sampling provides a tissue fragment of the cyst wall and has been proposed to improve the diagnostic yield. In addition to cytologic evaluation of cyst fluid obtained through FNA, TTN microforceps biopsy provide tissue specimens for better histopathologic evaluation through greater tissue specimen size and visualization of the histologic milieu, thereby increasing the diagnostic yield. This is similar to improved yield reported with histopathological evaluation of core biopsies obtained with EUS-guided fine needle biopsy (FNB) compared to FNA cytology alone for solid pancreatic lesions [54]. In our study, diagnostic yield and accuracy were significantly improved when compared to traditional FNA sampling with an OR $4.79(95 \% \mathrm{Cl}: 1.52$ to $15.06 ; P=$ $0.007)$ and $\mathrm{OR} 8.69(95 \% \mathrm{Cl}, 1.12$ to $67.12 ; P=0.038)$, respectively.

Specific limitations to this study include low-to-moderate heterogeneity of included studies. Despite this, studies included differences in patient population, varied definition EUSguided sampling techniques (i.e., suction vs slow-pull FNA, toand-fro movements, number or biopsies, number of passes, varied location of lesions and histopathology, as well as interoperator procedural capability and familiarity). Furthermore, this study included only one prospective observational study with no randomized trials included in this analysis. This lack of randomized trials may introduce a confounding bias as the main methodological problem in observational studies is the internal validity with lack of adjusted estimates in the systematic review and meta-analysis [55]. Publication bias was also assessed and present in this meta-analysis; however, correction of such did not significantly alter our findings given overlapping confidence intervals. Additionally, this microforceps device is not readily available at all centers with results likely reflecting centers with expertise. A concern with many innovative endoscopic procedures or techniques is the learning curve or clinical expertise needed to perform an effective procedure; however, given the design allowing for passage through a traditional 19gauge needle, this device may be easily adopted at centers with expertise in FNA of pancreatic lesions.

Despite these limitations, this study has several strengths. Most importantly, this systematic review and meta-analysis summarizes all available data to date to evaluate the feasibility, effectiveness, and rate of adverse events of TTN microforceps. Several studies were excluded upon in-depth evaluation of the literature due to overlapping enrollment periods or concern for duplication of patient data given certain multi-center studies [56-59]. Although low-to-moderate-heterogeneity was noted for some variables in our meta-analysis which is not surprising given the cumulative nature of reporting results, this is not sur- 

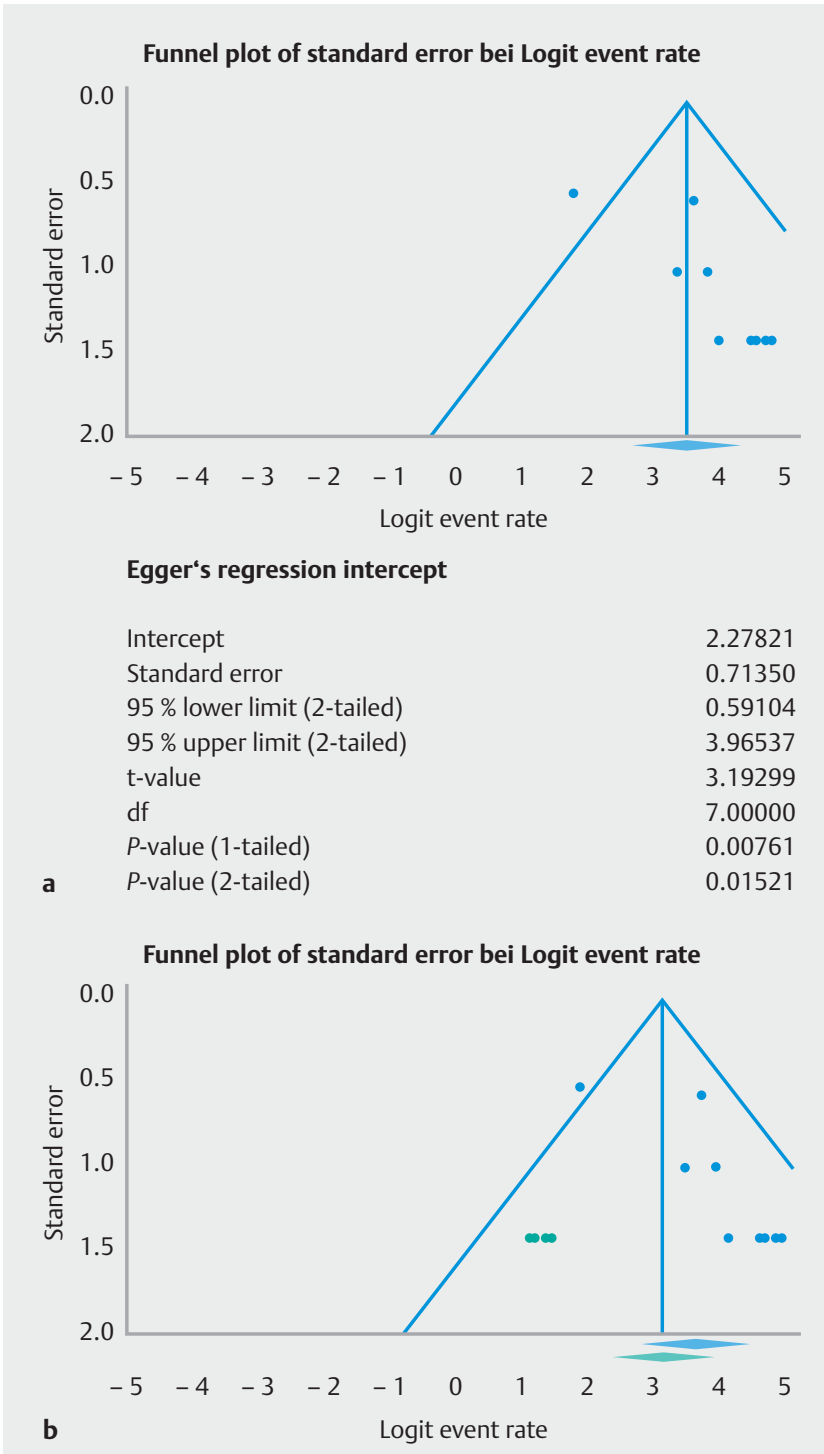

- Fig. 5 a Funnel plot of publication bias and eggers regression test for included studies to assess through-the-needle microforceps biopsies of pancreatic cystic lesions. $\mathbf{b}$ Funnel plot of publication bias with Duval and Tweedie's trim and fill method.

prising given a novel technique. Use of microceps biopsy may help to overcome limited cellularity of the EUS-guided cyst fluid aspiration and traditional cytology [60].

\section{Conclusion}

Overall, the TTN microceps biopsy device was demonstrated to be an effective modality for sampling of PCLs, with increased diagnostic yield compared to traditional FNA. To these authors' knowledge, this is the first systematic review and meta-analysis to summarize TTN microforceps biopsies for the diagnosis of PCLs. With future research, use of TTN may be incorporated into future guidelines though their exact role and indication remain less established at this time. While further prospective studies are needed prior to routine clinical implementation, we hope these findings provide an important step forward in future research and impact clinical decision making among advanced endoscopists.

\section{Competing interests}

Dr. Rustagi is a consultant for Boston Scientific.

\section{References}

[1] de Jong K, Nio CY, Hermans J] et al. High prevalence of pancreatic cysts detected by screening magnetic resonance imaging examinations. Clin Gastroenterol Hepatol 2010; 8: 806-811

[2] Laffan TA, Horton KM, Klein AP et al. Prevalence of unsuspected pancreatic cysts on MDCT. AJR Am J Roentgenol 2008; 191: 802-807

[3] Bosman FT, Carneiro F, Hruban RH et al. WHO classification of tumours of the digestive system. 4th ed. Lyon: International Agency for Research on Cancer; 2010

[4] Elta GH, Enestvedt BK, Sauer BG et al. ACG Clinical Guideline: Diagnosis and Management of Pancreatic Cysts. Am J Gastroenterol 2018; 113: 464-479

[5] Khan MA, Grimm IS, Ali B et al. A meta-analysis of endoscopic ultrasound-fine-needle aspiration compared to endoscopic ultrasoundfine-needle biopsy: diagnostic yield and the value of onsite cytopathological assessment. Endosc Int Open 2017; 5: E363-E75

[6] Vege SS, Ziring B, Jain R et al. American Gastroenterological Association institute guideline on the diagnosis and management of asymptomatic neoplastic pancreatic cysts. Gastroenterology 2015; 148: 819-822; quize12-3

[7] Del Chiaro M, Verbeke C, Salvia R et al. European experts consensus statement on cystic tumours of the pancreas. Dig Liver Dis 2013; 45 : 703-711

[8] Tanaka M, Fernandez-del Castillo C, Adsay V et al. International consensus guidelines 2012 for the management of IPMN and MCN of the pancreas. Pancreatology 2012; 12: 183-197

[9] Rogart JN, Loren DE, Singu BS et al. Cyst wall puncture and aspiration during EUS-guided fine needle aspiration may increase the diagnostic yield of mucinous cysts of the pancreas. J Clin Gastroenterol 2011; 45: 164-169

[10] Muthusamy VR, Chandrasekhara V. ASGE Standard of Practice Committee. et al. The role of endoscopy in the diagnosis and treatment of cystic pancreatic neoplasms. Gastrointest Endosc 2016; 84: 1-9

[11] Liberati A, Altman DG, Tetzlaff ] et al. The PRISMA statement for reporting systematic reviews and meta-analyses of studies that evaluate health care interventions: explanation and elaboration. Annals Int Med 2009; 151: W65-W94

[12] Stroup DF, Berlin JA, Morton SC et al. Meta-analysis of observational studies in epidemiology: a proposal for reporting. Meta-analysis Of Observational Studies in Epidemiology (MOOSE) group. JAMA 2000; 283: 2008-2012

[13] Cotton PB, Eisen GM, Aabakken L et al. A lexicon for endoscopic adverse events: report of an ASGE workshop. Gastrointest Endosc 2010; 71: 446-454

[14] DerSimonian R, Laird N. Meta-analysis in clinical trials. Controlled Clin Trials 1986; 7: 177-188

[15] Stuart A, Ord JK. Kendall's Advanced Theory of Statistics. 6th ed. London: Edward Arnold; 1994

[16] Riley RD, Higgins JPT, Deeks JJ. Research methods \& reporting: interpretation of random effects meta-analyses. Br Med J 2011; 342: d549 
[17] Higgins JP, Thompson SG, Deeks JJ et al. Measuring inconsistency in meta-analyses. BMJ 2003; 327: 557-560

[18] Overton RC. A comparison of fixed-effects and mixed (random-effects) models for meta-analysis tests of moderator variable effects. Psycholog Methods 1998; 3: 354-379

[19] Wells G, Shea B et al. The Newcastle-Ottawa Scale (NOS) for assessing the quality of nonrandomised studies in meta-analysis. 3rd Symposium on Systematic Reviews: Beyond the Basics; July 3-5 2000. Oxford: 2000: Available at (Accessed 24 November 2019): http://www. ohri.ca/programs/clinical_epidemiology/oxford.asp

[20] Higgins JPT, Altman DG, Sterne JAC. Cochrane Handbook for Systematic Reviews of Interventions. In: Higgins JPT Available from: www.cochranehandbook.com

[21] Borenstein M, Hedges LV, Higgins JP et al. Introduction to meta-analysis. Hoboken: John Wiley \& Sons; 2011

[22] Borenstein M, Higgins JP, Hedges LV et al. Basics of meta-analysis: I(2) is not an absolute measure of heterogeneity. Res Synth Methods 2017; 8: 5-18

[23] Egger M, Davey Smith G, Schneider M et al. Bias in meta-analysis detected by a simple, graphical test. BMJ 1997; 315: 629-634

[24] Easterbrook PJ, Berlin JA, Gopalan R et al. Publication bias in clinical research. Lancet 1991; 337: 867-672

[25] Duval S, Tweedie R. Trim and fill: a simple funnel-plot-based method of testing and adjusting for publication bias in metaanalysis. Biometrics 2000; 56: 455-463

[26] Kovacevic B, Karstensen JG, Havre RF et al. Initial experience with EUSguided microbiopsy forceps in diagnosing pancreatic cystic lesions: A multicenter feasibility study (with video). Endosc Ultrasound 2018; 7: 383-388

[27] Mittal C, Obuch JC, Hammad H et al. Technical feasibility, diagnostic yield, and safety of microforceps biopsies during EUS evaluation of pancreatic cystic lesions (with video). Gastrointest Endosc 2018; 87: 1263-1269

[28] Yang D, Samarasena JB, Jamil LH et al. Endoscopic ultrasound-guided through-the-needle microforceps biopsy in the evaluation of pancreatic cystic lesions: a multicenter study. Endosc Int Open 2018; 6: E1423-E30

[29] Zhang ML, Arpin RN, Brugge WR et al. Moray micro forceps biopsy improves the diagnosis of specific pancreatic cysts. Cancer Cytopathol 2018; 126: 414-420

[30] Cheesman AR, Zhu H, Liao X et al. Impact of EUS-guided microforceps biopsy and needle-based confocal laser endomicroscopy on the diagnostic yield and clinical management of pancreatic cystic lesions. Gastrointest Endosc 2020; 91: 1095-1104

[31] Crino SF, Bernardoni L, Brozzi L et al. Association between macroscopically visible tissue samples and diagnostic accuracy of EUS-guided through-the-needle microforceps biopsy sampling of pancreatic cystic lesions. Gastrointest Endosc 2019; 90: 933-943

[32] Hashimoto R, Lee JG, Chang KJ et al. Endoscopic ultrasound-throughthe-needle biopsy in pancreatic cystic lesions: A large single center experience. World J Gastrointest Endosc 2019; 11: 531-540

[33] Robles-Medrandra C, Olmos JI, Oleas R et al. EUS-Through-the-needle technologies in the diagnosis and malignancy detection of pancreatic cysts: a comparative study between different technologies. Gastrointest Endosc 2019; 89: AB608-A609

[34] Vestrup Rift C, Melchior LC, Kovacevic B et al. Next-generation sequencing of endoscopic ultrasound guided microbiopsies from pancreatic cystic neoplasms. Histopathology 2019; 75: 767-771

[35] Wilen J, Visrodia K, Lan G et al. The feasibility and value of cyst wall biopsy using micro forceps in the diagnosis of pancreatic cysts. Gastrointest Endosc 2019; 89: AB605

[36] Yang D, Trindade AJ, Yachimski P et al. Histologic Analysis of endoscopic ultrasound-guided through the needle microforceps biopsies accurately identifies mucinous pancreas cysts. Clin Gastroenterol Hepatol 2019; 17: 1587-1596

[37] Zerboni G, Signoretti M, Crippa S et al. Systematic review and metaanalysis: Prevalence of incidentally detected pancreatic cystic lesions in asymptomatic individuals. Pancreatology 2019; 19: 2-9

[38] Farrell J]. Prevalence, diagnosis and management of pancreatic cystic neoplasms: current status and future directions. Gut Liver 2015; 9: 571-589

[39] Buscarini E et al. Italian Association of Hospital Gastroenterologists and Endoscopists, Italian Association for the Study of the Pancreas. Italian consensus guidelines for the diagnostic work-up and follow-up of cystic pancreatic neoplasms. Dig Liver Dis 2014; 46: 479-493

[40] de Pretis N, Mukewar S, Aryal-Khanal A et al. Pancreatic cysts: Diagnostic accuracy and risk of inappropriate resections. Pancreatology 2017; 17: 267-272

[41] Larino-Noia J, Iglesias-Garcia J, de la Iglesia-Garcia D et al. EUS-FNA in cystic pancreatic lesions: Where are we now and where are we headed in the future? Endosc Ultrasound 2018; 7: 102-109

[42] Brugge WR, Lewandrowski K, Lee-Lewandrowski E et al. Diagnosis of pancreatic cystic neoplasms: a report of the cooperative pancreatic cyst study. Gastroenterology 2004; 126: 1330-1336

[43] Cizginer S, Turner BG, Bilge AR et al. Cyst fluid carcinoembryonic antigen is an accurate diagnostic marker of pancreatic mucinous cysts. Pancreas 2011; 40: 1024-1028

[44] Gaddam S, Ge PS, Keach JW et al. Suboptimal accuracy of carcinoembryonic antigen in differentiation of mucinous and nonmucinous pancreatic cysts: results of a large multicenter study. Gastrointest Endosc 2015; 82: 1060-1069

[45] Khalid A, Zahid M, Finkelstein SD et al. Pancreatic cyst fluid DNA analysis in evaluating pancreatic cysts: a report of the PANDA study. Gastrointest Endosc 2009; 69: 1095-1102

[46] Nakai Y, Iwashita T, Park DH et al. Diagnosis of pancreatic cysts: EUSguided, through-the-needle confocal laser-induced endomicroscopy and cystoscopy trial: DETECT study. Gastrointest Endosc 2015; 81: 1204-1214

[47] Singhi AD, Nikiforova MN, Fasanella KE et al. Preoperative GNAS and KRAS testing in the diagnosis of pancreatic mucinous cysts. Clin Cancer Res 2014; 20: 4381-4389

[48] Iglesias-Garcia J, Larino-Noia J, Abdulkader I et al. Rapid on-site evaluation of endoscopic-ultrasound-guided fine-needle aspiration diagnosis of pancreatic masses. World J Gastroenterol 2014; 20: 94519457

[49] Klapman JB, Logrono R, Dye CE et al. Clinical impact of on-site cytopathology interpretation on endoscopic ultrasound-guided fine needle aspiration. Am J Gastroenterol 2003; 98: 1289-1294

[50] European Study Group on Cystic Tumours of the Pancreas. European evidence-based guidelines on pancreatic cystic neoplasms. Gut 2018; 67: 789-804

[51] Aparicio JR, Martinez J, Niveiro M et al. Direct intracystic biopsy and pancreatic cystoscopy through a 19-gauge needle EUS (with videos). Gastrointest Endosc 2010; 72: 1285-1288

[52] Barresi L, Tarantino I, Ligresti D et al. A new tissue acquisition technique in pancreatic cystic neoplasm: endoscopic ultrasound-guided through-the-needle forceps biopsy. Endoscopy 2015; 47: (Suppl. 01): E297-E298

[53] Shakhatreh MH, Naini SR, Brijbassie AA et al. Use of a novel throughthe-needle biopsy forceps in endoscopic ultrasound. Endosc Int Open 2016; 4: E439-E442

[54] Iglesias-Garcia J, Dominguez-Munoz JE, Abdulkader I et al. Influence of on-site cytopathology evaluation on the diagnostic accuracy of endoscopic ultrasound-guided fine needle aspiration (EUS-FNA) of solid pancreatic masses. Am J Gastroenterol 2011; 106: 1705-1710 
[55] Faber T, Ravaud P, Riveros C et al. Meta-analyses including non-randomized studies of therapeutic interventions: a methodological review. BMC Med Res Methodol 2016; 16: 35

[56] Basar O, Yuksel O, Yang DJ et al. Feasibility and safety of microforceps biopsy in the diagnosis of pancreatic cysts. Gastrointest Endosc 2018; 88: $79-86$

[57] Kovacevic B, Klausen P, Hasselby JP et al. A novel endoscopic ultrasound-guided through-the-needle microbiopsy procedure improves diagnosis of pancreatic cystic lesions. Endoscopy 2018; 50: 11051111
[58] Barresi L, Crino SF, Fabbri C et al. Endoscopic ultrasound-through-theneedle biopsy in pancreatic cystic lesions: A multicenter study. Dig Endosc 2018; 30: 760-770

[59] Samarasena J, Yu A, Lee D et al. EUS-guided through-the-needle biopsy for pancreatic cystic lesions. VideoGIE 2019; 4: 436-439

[60] Kohoutova D, Zar S, Repak R et al. Pancreatic Cysts: Diagnostic role of EUS-guided microforceps biopsy and confocal laser endomicroscopy. Gastroenterol Res Pract 2019; 2019: 3431048 